\title{
Oscillator strengths for transitions in C-like ions between K XIV and Mn XX`
}

\author{
K. M. Aggarwal ${ }^{1}$, F. P. Keenan ${ }^{1}$, and A. Z. Msezane $^{2}$ \\ ${ }^{1}$ Department of Pure and Applied Physics, The Queen's University of Belfast, Belfast BT7 1NN, Northern Ireland, UK \\ 2 Center for Theoretical Studies of Physical Systems, Clark Atlanta University, Atlanta, Ga 30314, USA
}

Received 5 December 2002 / Accepted 21 January 2003

\begin{abstract}
Energy levels and oscillator strengths (transition probabilities) have been calculated for transitions among 46 finestructure levels of the $\left(1 s^{2}\right) 2 s^{2} 2 p^{2}, 2 s 2 p^{3}, 2 p^{4}, 2 s^{2} 2 p 3 s, 2 s^{2} 2 p 3 p$ and $2 s^{2} 2 p 3 d$ configurations of C-like K XIV, Sc XVI, Ti XVII, V XVIII, Cr XIX and Mn XX using the GRASP code. Configuration interaction and relativistic effects have been included while generating the wavefunctions. Calculated values of energy levels agree within $3 \%$ with the experimentally compiled results, and the length and velocity forms of oscillator strengths agree within $20 \%$ for a majority of allowed transitions.
\end{abstract}

Key words. atomic data - atomic processes

\section{Introduction}

This is a continuation of our efforts to generate atomic data (energy levels, oscillator strengths, radiative rates, collision strengths, and excitation rates) for C-like ions. Several papers have been published over the past two decades reporting such data, particularly for energy levels and radiative rates. To summarise, these papers are by Aggarwal et al. (1997a) for O III, Aggarwal et al. (1997b) for Ca XV and Fe XXI, Aggarwal (1998) for Ne V, Mg VII, Si IX and S XI, and Aggarwal et al. (2001) for F IV, Na VI, Al VIII, P X, Cl XII and Ar XIII. In the present paper we report similar data for six other C-like ions, namely K XIV, Sc XVI, Ti XVII, V XVIII, Cr XIX and Mn XX. This work completes the sequence for ions with nuclear charges $8 \leq Z \leq 26$.

Emission lines of C-like ions are highly useful as diagnostics of solar, astrophysical and fusion plasmas, the interpretation of which requires the knowledge of atomic parameters. Emission lines of the ions for which we present data in this paper have already been observed (Kelly 1987). Therefore, a few researchers in the past have reported atomic data for $\mathrm{C}$-like ions. However, most of the calculations have been confined to the lowest 20 levels of the $\left(1 \mathrm{~s}^{2}\right) 2 \mathrm{~s}^{2} 2 \mathrm{p}^{2}, 2 \mathrm{~s} 2 \mathrm{p}^{3}$ and $2 \mathrm{p}^{4}$ configurations. Similar data for transitions among the levels of the $2 s^{2} 2 p 3 s, 2 s^{2} 2 p 3 p$ and $2 s^{2} 2 p 3 d$ configurations, which mostly lie in the X-ray and EUV regions, are equally important, especially for the modeling of astrophysical and laboratory plasmas, and therefore have become increasingly in demand. Fawcett (1987)

\footnotetext{
Send offprint requests to: K. M. Aggarwal,

e-mail: K.Aggarwal@qub.ac.uk

* Tables 4 to 9 are only available in electronic form at http://www. edpsciences.org
}

has included levels of the $2 s^{2} 2 p 3 s$ and $2 s^{2} 2 p 3 d$ configurations in his calculations, whereas Zhang \& Sampson (1996, 1997) have also included levels of the $2 s^{2} 2 p 3 p$ configuration. Both Fawcett and Zhang \& Sampson have included configuration interaction (CI) and relativistic effects (RE) in their calculations, as these are equally important particularly for the ions under discussion here. However, atomic data have been reported for a few transitions only, and therefore, our aim is to report results of oscillator strengths and radiative rates for all allowed transitions among 46 fine-structure levels of the $\left(1 \mathrm{~s}^{2}\right)$ $2 \mathrm{~s}^{2} 2 \mathrm{p}^{2}, 2 \mathrm{~s} 2 \mathrm{p}^{3}, 2 \mathrm{p}^{4}, 2 \mathrm{~s}^{2} 2 \mathrm{p} 3 \mathrm{~s}, 2 \mathrm{~s}^{2} 2 \mathrm{p} 3 \mathrm{p}$ and $2 \mathrm{~s}^{2} 2 \mathrm{p} 3 \mathrm{~d}$ configurations. Additionally, we find that there is scope for improvement over the earlier results, because the inclusion of CI in these calculations is rather restricted.

\section{Calculations}

In almost all our previous work referred to above, we have adopted the CIV3 program of Hibbert (1975) to perform our calculations for energy levels and radiative rates. However, in the cases of $\mathrm{Ca} \mathrm{XV}$ and Fe XXI we also performed an additional set of calculations employing the GRASP code of Dyall et al. (1989), as relativistic effects are very important for these and other ions with similar or higher $Z$. The inclusion of $\mathrm{CI}$ in our GRASP calculations was restricted to that within the basic (above listed six) configurations only. On the other hand, the CIV3 calculations considered more elaborate CI including external orbitals, but included only one-body relativistic operators in a Breit-Pauli approximation, namely spin-orbit, spinother-orbit, spin-spin, Darwin and mass correction terms. In the present work we find this approach of $L S J$ coupling scheme to be inadequate, as both $\mathrm{CI}$ and RE are equally important 
for the ions under consideration here, and therefore we have adopted the fully relativistic GRASP code (which is based on the $j j$ coupling scheme), but with inclusion of larger CI. To be specific, CI has been included among the $1 \mathrm{~s}^{2} 2 \mathrm{~s}^{2} 2 \mathrm{p}^{2}, 2 \mathrm{~s} 2 \mathrm{p}^{3}$, $2 \mathrm{p}^{4}, 2 \mathrm{~s}^{2} 2 \mathrm{p} 3 \ell, 2 \mathrm{~s} 2 \mathrm{p}^{2} 3 \ell, 2 \mathrm{p}^{3} 3 \ell$ and $2 \mathrm{~s}^{2} 3 \ell^{2}$ configurations. These 15 configurations give rise to 251 fine-structure levels, but results for energy levels and radiative rates are reported for those among the lowest 46 levels of the first six configurations only. The Breit and QED corrections have been included in the calculations, and the option of EAL (extended average level) has been adopted, as in all our earlier works. In this type of calculation, a weighted trace of the Hamiltonian matrix is minimized, which yields a compromise set of orbitals describing closely lying states with moderate accuracy.

\section{Results and discussion}

In Tables 1-3 we list the energy levels arising from the $\left(1 s^{2}\right) 2 s^{2} 2 p^{2}, 2 s 2 p^{3}, 2 p^{4}, 2 s^{2} 2 p 3 s, 2 s^{2} 2 p 3 p$ and $2 s^{2} 2 p 3 d$ configurations of $\mathrm{C}$-like ions, and compare our threshold energies for K XIV, Sc XVI, Ti XVII, V XVIII, Cr XIX and $\mathrm{Mn}$ XX with the experimental values compiled by NIST (National Institute for Standards and Technology: WWW. physics.nist.gov/PhysRefData). Since the ordering of the energy levels is not the same for all C-like ions, the levels are listed in Tables 1-3 for each ion. The first column of each table provides an index for the corresponding level. However, it may be noted that the experimental values are not available for all levels, and the accuracy for some of these, especially those among the $2 s^{2} 2 \mathrm{p} 3 \mathrm{~s}, 2 \mathrm{~s}^{2} 2 \mathrm{p} 3 \mathrm{p}$ and $2 \mathrm{~s}^{2} 2 \mathrm{p} 3 \mathrm{~d}$ configurations, is not very high. The theoretical values available in the literature are mostly confined to the lowest 20 levels, but corresponding results involving some of the higher excited levels have been reported by Fawcett (1987) and Zhang \& Sampson (1997) in the form of transition wavelengths.

The agreement of our level energies with the experimental ones is excellent (within 3\%) for all levels and for all ions. It may be noted that the energy order of the theoretical and experimental energy levels is slightly different in a few instances; see, for example, the highest two levels of K XIV and Sc XVI. However, the energy differences for such levels are very small ( $\leq 0.03 \mathrm{Ryd})$. This problem of energy order is frequently encountered in all theoretical work, especially when the energy separation of the levels is very small. Furthermore, in the absence of definitive experimental level energies in most of the cases, a correct energy order cannot be confirmed. In any event, it does not affect the computed values of the oscillator strengths and collision strengths.

In Tables 4-9 we present our oscillator strengths and radiative rates (in length form only) for allowed transitions in $\mathrm{K} X I V$, Sc XVI, Ti XVII, V XVIII, Cr XIX and Mn XX. The indices used to represent the levels of a transition are already given in Tables 1-3. Since the indices of the various levels are not the same for all the ions, we have included in these tables the shorter forms of the lower and upper levels of a transition. This should facilitate their identification.

The other values available in the literature for oscillator strengths ( $f$-values) and radiative rates ( $A$-values), apart from those of Fawcett (1987), are of Zhang \& Sampson (1996) for a few transitions among the lowest 20 levels. Their results are not only the most recent for the present ions, but are also similar and comparable to our present calculations, as they too have adopted the GRASP code, but have restricted the inclusion of CI among the first three configurations only, i.e. $2 s^{2} 2 p^{2}, 2 s 2 p^{3}$ and $2 p^{4}$. Zhang \& Sampson (1997) have performed a larger calculation including the levels of the $2 \mathrm{~s}^{2} 2 \mathrm{p} 3 \mathrm{~s}$, $2 s^{2} 2 p 3 p$ and $2 s^{2} 2 p 3 d$ configurations, but have not reported any data for the ions under discussion here. However, a comparison between our present and their earlier results for oscillator strengths shows agreement within $20 \%$ for the available common transitions for all ions. This is an expected result considering the limited amount of CI included by them.

Among the higher excited levels, the only results available in the literature are by Fawcett (1987), who has adopted the Hartree-Fock relativistic (HFR) code of Cowan (1981). In Table 10 we compare our values of oscillator strengths for a few representative transitions with his HFR results. The indices used to represent a transition correspond to those of K XIV, but a shorter form of the transitions has been included in Col. 3 in order to facilitate an easy identification of the same transition in different ions. Generally, our GRASP and earlier HFR $f$-values agree within $10 \%$, but for a few transitions (such as 2-40 and 5-45) the differences are up to 30\%, and for the 2-45 $\left(2 \mathrm{~s}^{2} 2 \mathrm{p}^{2}{ }^{3} \mathrm{P}_{1}-2 \mathrm{~s}^{2} 2 \mathrm{p} 3 \mathrm{~d}^{1} \mathrm{P}_{1}^{\circ}\right)$ and 4-37 $\left(2 \mathrm{~s}^{2} 2 \mathrm{p}^{2}{ }^{1} \mathrm{D}_{2}-\right.$ $\left.2 \mathrm{~s}^{2} 2 \mathrm{p} 3 \mathrm{~d}^{1} \mathrm{D}_{2}^{\circ}\right)$ transitions, the two sets of results differ by a factor of two. However, the transitions for which differences between the two sets of calculations are significant are weaker transitions, whose $f$-values are comparatively small. These differences arise due to different amount of CI included in the calculations.

In general, inclusion of larger CI improves the wavefunctions, and hence leads to a more accurate determination of energy levels and radiative rates for a majority of transitions. However, occasionally it may also result in comparatively reduced accuracy for a few transitions, as has specifically been discussed by Aggarwal (1998) for transitions in Fe XXI. A further example of this is provided by the $2-45\left(2 \mathrm{~s}^{2} 2 \mathrm{p}^{2}{ }^{3} \mathrm{P}_{1}-\right.$ $2 \mathrm{~s}^{2} 2 \mathrm{p} 3 \mathrm{~d}{ }^{1} \mathrm{P}_{1}^{\circ}$ ) transition in Sc XVI, whose $f$-value of $7.545 \times$ $10^{-4}$ is incongrous with the corresponding $f$-values of other ions as shown in Table 10. Our calculations with 6, 12, 15, 16,19 and 20 configurations yield the $f$-values $3.807 \times 10^{-3}$, $1.535 \times 10^{-4}, 7.545 \times 10^{-4}, 5.168 \times 10^{-3}, 5.184 \times 10^{-3}$ and $5.130 \times 10^{-3}$, respectively. Clearly, the $f$-value for this transition should be $\sim 10^{-3}$, and the presently reported result of $7.545 \times 10^{-4}$ is underestimated by a factor of seven. There could be some other similar examples, but a majority of transitions are unaffected by such inconsistencies.

The accuracy of the calculated $f$ - or $A$-values is generally (but not conclusively) determined by the agreement between their length and velocity forms. In any large calculation involving a large number of transitions, as in the present work, the agreement between the two forms for all the transitions is never satisfactory. Weak transitions $(f<0.10)$ being sensitive to mixing often show large variations, which can be over an order of magnitude. However, these transitions are not very important, in general. For transitions whose $f$-values are 
Table 1. Target levels of K XIV and Sc XVI, and their threshold energies (in Ryd).

\begin{tabular}{|c|c|c|c|c|c|c|c|c|}
\hline \multirow[b]{2}{*}{ Index } & \multicolumn{4}{|c|}{ K XIV } & \multicolumn{4}{|c|}{ Sc XVI } \\
\hline & Configuration & Level & Expt. $^{a}$ & GRASP & Configuration & Level & Expt. $^{a}$ & GRASP \\
\hline $1 \ldots \ldots$ & $1 s^{2} 2 s^{2} 2 p^{2}$ & ${ }^{3} \mathrm{P}_{0}$ & 0.0000 & 0.0000 & $1 s^{2} 2 s^{2} 2 p^{2}$ & ${ }^{3} \mathrm{P}_{0}$ & 0.0000 & 0.0000 \\
\hline $2 \ldots \ldots$ & $1 s^{2} 2 s^{2} 2 p^{2}$ & ${ }^{3} \mathrm{P}_{1}$ & 0.1206 & 0.1196 & $1 s^{2} 2 s^{2} 2 p^{2}$ & ${ }^{3} \mathrm{P}_{1}$ & 0.2092 & 0.2076 \\
\hline $3 \ldots \ldots$ & $1 s^{2} 2 s^{2} 2 p^{2}$ & ${ }^{3} \mathrm{P}_{2}$ & 0.2572 & 0.2564 & $1 s^{2} 2 s^{2} 2 p^{2}$ & ${ }^{3} \mathrm{P}_{2}$ & 0.4103 & 0.4099 \\
\hline $4 \ldots \ldots$ & $1 \mathrm{~s}^{2} 2 \mathrm{~s}^{2} 2 \mathrm{p}^{2}$ & ${ }^{1} \mathrm{D}_{2}$ & 0.8740 & 0.9017 & $1 s^{2} 2 s^{2} 2 p^{2}$ & ${ }^{1} \mathrm{D}_{2}$ & 1.1241 & 1.1495 \\
\hline $5 \ldots \ldots$ & $1 s^{2} 2 s^{2} 2 p^{2}$ & ${ }^{1} \mathrm{~S}_{0}$ & 1.6304 & 1.6390 & $1 s^{2} 2 s^{2} 2 p^{2}$ & ${ }^{1} \mathrm{~S}_{0}$ & 1.9931 & 1.9996 \\
\hline $6 \ldots \ldots$ & $1 \mathrm{~s}^{2} 2 \mathrm{~s} 2 \mathrm{p}^{3}$ & ${ }^{5} \mathrm{~S}_{2}^{\circ}$ & 2.2840 & 2.2160 & $1 \mathrm{~s}^{2} 2 \mathrm{~s} 2 \mathrm{p}^{3}$ & ${ }^{5} \mathrm{~S}_{2}^{\circ}$ & 2.7460 & 2.6986 \\
\hline $7 \ldots \ldots$ & $1 \mathrm{~s}^{2} 2 \mathrm{~s} 2 \mathrm{p}^{3}$ & ${ }^{3} \mathrm{D}_{2}^{\circ}$ & 4.1805 & 4.2224 & $1 s^{2} 2 s 2 p^{3}$ & ${ }^{3} \mathrm{D}_{2}^{\circ}$ & 4.8899 & 4.9325 \\
\hline $8 \ldots \ldots$ & $1 s^{2} 2 s 2 p^{3}$ & ${ }^{3} \mathrm{D}^{\circ}{ }_{1}$ & 4.1873 & 4.2293 & $1 s^{2} 2 s 2 p^{3}$ & ${ }^{3} \mathrm{D}^{\circ}{ }_{1}$ & 4.9000 & 4.9433 \\
\hline $9 \ldots \ldots$ & $1 \mathrm{~s}^{2} 2 \mathrm{~s} 2 \mathrm{p}^{3}$ & ${ }^{3} \mathrm{D}_{3}^{\circ}$ & 4.2010 & 4.2413 & $1 \mathrm{~s}^{2} 2 \mathrm{~s} 2 \mathrm{p}^{3}$ & ${ }^{3} \mathrm{D}_{3}^{\circ}$ & 4.9393 & 4.9800 \\
\hline $10 \ldots \ldots$ & $1 s^{2} 2 s 2 p^{3}$ & ${ }^{3} \mathrm{P}_{0}^{\circ}$ & 4.8972 & 4.9500 & $1 s^{2} 2 s 2 p^{3}$ & ${ }^{3} \mathrm{P}_{0}^{\circ}$ & 5.7280 & 5.7816 \\
\hline $11 \ldots \ldots$ & $1 s^{2} 2 s 2 p^{3}$ & ${ }^{3} \mathrm{P}^{\circ}{ }_{1}$ & 4.9029 & 4.9556 & $1 s^{2} 2 s 2 p^{3}$ & ${ }^{3} \mathrm{P}_{1}^{\circ}$ & 5.7432 & 5.7965 \\
\hline $12 \ldots \ldots$ & $1 s^{2} 2 s 2 p^{3}$ & ${ }^{3} \mathrm{P}_{2}^{\circ}$ & 4.9203 & 4.9722 & $1 s^{2} 2 s 2 p^{3}$ & ${ }^{3} \mathrm{P}_{2}^{\circ}$ & 5.7813 & 5.8332 \\
\hline $13 \ldots \ldots$ & $1 s^{2} 2 s 2 p^{3}$ & ${ }^{3} \mathrm{~S}^{\circ}{ }_{1}$ & 6.1757 & 6.2936 & $1 s^{2} 2 s 2 p^{3}$ & ${ }^{3} \mathrm{~S}^{\circ}{ }_{1}$ & 7.1293 & 7.2478 \\
\hline $14 \ldots \ldots$ & $1 \mathrm{~s}^{2} 2 \mathrm{~s} 2 \mathrm{p}^{3}$ & ${ }^{1} \mathrm{D}_{2}^{\circ}$ & 6.1643 & 6.2994 & $1 s^{2} 2 s 2 p^{3}$ & ${ }^{1} \mathrm{D}_{2}^{\circ}$ & 7.1601 & 7.2941 \\
\hline $15 \ldots \ldots$ & $1 s^{2} 2 s 2 p^{3}$ & ${ }^{1} \mathrm{P}^{\circ}{ }_{1}$ & 6.8805 & 7.0246 & $1 s^{2} 2 s 2 p^{3}$ & ${ }^{1} \mathrm{P}^{\circ}{ }_{1}$ & 7.9900 & 8.1345 \\
\hline $16 \ldots \ldots$ & $1 s^{2} 2 p^{4}$ & ${ }^{3} \mathrm{P}_{2}$ & 9.3869 & 9.5251 & $1 s^{2} 2 p^{4}$ & ${ }^{3} \mathrm{P}_{2}$ & 10.824 & 10.9652 \\
\hline $17 \ldots \ldots$ & $1 s^{2} 2 p^{4}$ & ${ }^{3} \mathrm{P}_{1}$ & 9.5740 & 9.7092 & $1 s^{2} 2 p^{4}$ & ${ }^{3} \mathrm{P}_{1}$ & 11.129 & 11.2655 \\
\hline $18 \ldots \ldots$ & $1 s^{2} 2 p^{4}$ & ${ }^{3} \mathrm{P}_{0}$ & 9.6248 & 9.7608 & $1 s^{2} 2 p^{4}$ & ${ }^{3} \mathrm{P}_{0}$ & 11.188 & 11.3278 \\
\hline $19 \ldots \ldots$ & $1 s^{2} 2 p^{4}$ & ${ }^{1} \mathrm{D}_{2}$ & 10.104 & 10.2920 & $1 s^{2} 2 p^{4}$ & ${ }^{1} \mathrm{D}_{2}$ & 11.714 & 11.9019 \\
\hline $20 \ldots \ldots$ & $1 s^{2} 2 p^{4}$ & ${ }^{1} \mathrm{~S}_{0}$ & 11.435 & 11.6672 & $1 s^{2} 2 p^{4}$ & ${ }^{1} \mathrm{~S}_{0}$ & 13.226 & 13.4574 \\
\hline $21 \ldots \ldots$ & $1 s^{2} 2 s^{2} 2 p 3 s$ & ${ }^{3} \mathrm{P}_{0}^{\circ}$ & $\ldots \ldots$. & 32.6831 & $1 s^{2} 2 s^{2} 2 p 3 s$ & ${ }^{3} \mathrm{P}_{0}^{\circ}$ & $\ldots \ldots$ & 41.8443 \\
\hline $22 \ldots \ldots$ & $1 s^{2} 2 s^{2} 2 p 3 s$ & ${ }^{3} \mathrm{P}^{\circ}{ }_{1}$ & ...... & 32.7250 & $1 s^{2} 2 s^{2} 2 p 3 s$ & ${ }^{3} \mathrm{P}^{\circ}{ }_{1}$ & 41.970 & 41.8981 \\
\hline $23 \ldots \ldots$ & $1 s^{2} 2 s^{2} 2 p 3 s$ & ${ }^{3} \mathrm{P}_{2}^{\circ}$ & ....... & 32.9437 & $1 s^{2} 2 s^{2} 2 p 3 s$ & ${ }^{3} \mathrm{P}_{2}^{\circ}$ & 42.330 & 42.2547 \\
\hline $24 \ldots \ldots$ & $1 s^{2} 2 s^{2} 2 p 3 s$ & ${ }^{1} \mathrm{P}^{\circ}{ }_{1}$ & $\ldots \ldots$ & 33.1109 & $1 s^{2} 2 s^{2} 2 p 3 s$ & ${ }^{1} \mathrm{P}^{\circ}{ }_{1}$ & 42.490 & 42.4381 \\
\hline $25 \ldots \ldots$ & $1 s^{2} 2 s^{2} 2 p 3 p$ & ${ }^{1} \mathrm{P}_{1}$ & $\ldots \ldots$. & 33.7630 & $1 s^{2} 2 s^{2} 2 p 3 p$ & ${ }^{3} \mathrm{D}_{1}$ & ....... & 43.0839 \\
\hline $26 \ldots \ldots$ & $1 s^{2} 2 s^{2} 2 p 3 p$ & ${ }^{3} \mathrm{D}_{1}$ & $\ldots \ldots$ & 33.9296 & $1 s^{2} 2 s^{2} 2 p 3 p$ & ${ }^{1} \mathrm{P}_{1}$ & $\ldots \ldots$ & 43.3261 \\
\hline $27 \ldots \ldots$ & $1 s^{2} 2 s^{2} 2 p 3 p$ & ${ }^{3} \mathrm{D}_{2}$ & $\ldots \ldots$ & 33.9419 & $1 s^{2} 2 s^{2} 2 p 3 p$ & ${ }^{3} \mathrm{D}_{2}$ & $\ldots \ldots$ & 43.3370 \\
\hline $28 \ldots \ldots$ & $1 s^{2} 2 s^{2} 2 p 3 p$ & ${ }^{3} \mathrm{P}_{0}$ & $\ldots \ldots$ & 34.1207 & $1 s^{2} 2 s^{2} 2 p 3 p$ & ${ }^{3} \mathrm{P}_{0}$ & $\ldots \ldots$ & 43.5175 \\
\hline $29 \ldots \ldots$ & $1 s^{2} 2 s^{2} 2 p 3 p$ & ${ }^{3} \mathrm{D}_{3}$ & $\ldots \ldots$ & 34.1253 & $1 s^{2} 2 s^{2} 2 p 3 p$ & ${ }^{3} \mathrm{P}_{1}$ & $\ldots \ldots$ & 43.6380 \\
\hline $30 \ldots \ldots$ & $1 s^{2} 2 s^{2} 2 p 3 p$ & ${ }^{3} S_{1}$ & $\ldots \ldots$. & 34.1557 & $1 s^{2} 2 s^{2} 2 p 3 p$ & ${ }^{3} \mathrm{D}_{3}$ & ....... & 43.6360 \\
\hline $31 \ldots \ldots$ & $1 s^{2} 2 s^{2} 2 p 3 p$ & ${ }^{3} \mathrm{P}_{1}$ & $\ldots \ldots$ & 34.2616 & $1 s^{2} 2 s^{2} 2 p 3 p$ & ${ }^{3} \mathrm{~S}_{1}$ & $\ldots \ldots$ & 43.7675 \\
\hline $32 \ldots \ldots$ & $1 s^{2} 2 s^{2} 2 p 3 p$ & ${ }^{3} \mathrm{P}_{2}$ & $\ldots \ldots$ & 34.3046 & $1 s^{2} 2 s^{2} 2 p 3 p$ & ${ }^{3} \mathrm{P}_{2}$ & ...... & 43.8172 \\
\hline $33 \ldots \ldots$ & $1 s^{2} 2 s^{2} 2 p 3 p$ & ${ }^{1} \mathrm{D}_{2}$ & ...... & 34.6637 & $1 s^{2} 2 s^{2} 2 p 3 p$ & ${ }^{1} D_{2}$ & ...... & 44.2242 \\
\hline $34 \ldots \ldots$ & $1 s^{2} 2 s^{2} 2 p 3 p$ & ${ }^{1} \mathrm{~S}_{0}$ & $\ldots \ldots$ & 35.0815 & $1 s^{2} 2 s^{2} 2 p 3 d$ & ${ }^{3} \mathrm{~F}^{\circ}{ }_{2}$ & 44.000 & 44.6449 \\
\hline $35 \ldots \ldots$ & $1 s^{2} 2 s^{2} 2 p 3 d$ & ${ }^{3} \mathrm{~F}_{2}^{\circ}$ & $\ldots \ldots$. & 35.1118 & $1 s^{2} 2 s^{2} 2 p 3 p$ & ${ }^{1} \mathrm{~S}_{0}$ & $\ldots \ldots$ & 44.6965 \\
\hline $36 \ldots \ldots$ & $1 s^{2} 2 s^{2} 2 p 3 d$ & ${ }^{3} \mathrm{~F}_{3}^{\circ}$ & 35.281 & 35.2249 & $1 s^{2} 2 s^{2} 2 p 3 d$ & ${ }^{3} \mathrm{~F}_{3}^{\circ}$ & $\ldots \ldots$ & 44.8108 \\
\hline $37 \ldots \ldots$ & $1 s^{2} 2 s^{2} 2 p 3 d$ & ${ }^{1} \mathrm{D}_{2}^{\circ}$ & $\ldots \ldots$ & 35.2784 & $1 s^{2} 2 s^{2} 2 p 3 d$ & ${ }^{1} \mathrm{D}_{2}^{\circ}$ & $\ldots \ldots$ & 44.8758 \\
\hline $38 \ldots \ldots$ & $1 s^{2} 2 s^{2} 2 p 3 d$ & ${ }^{3} \mathrm{~F}_{4}^{\circ}$ & $\ldots \ldots$. & 35.3805 & $1 s^{2} 2 s^{2} 2 p 3 d$ & ${ }^{3} \mathrm{D}^{\circ}{ }_{1}$ & 45.070 & 45.0389 \\
\hline $39 \ldots \ldots$ & $1 s^{2} 2 s^{2} 2 p 3 d$ & ${ }^{3} \mathrm{D}_{1}^{\circ}$ & $\ldots \ldots$ & 35.4403 & $1 s^{2} 2 s^{2} 2 p 3 d$ & ${ }^{3} \mathrm{~F}_{4}^{\circ}$ & $\ldots \ldots$ & 45.0710 \\
\hline $40 \ldots \ldots$ & $1 s^{2} 2 s^{2} 2 p 3 d$ & ${ }^{3} \mathrm{D}_{2}^{\circ}$ & 35.540 & 35.5013 & $1 s^{2} 2 s^{2} 2 p 3 d$ & ${ }^{3} \mathrm{D}_{2}^{\circ}$ & 45.100 & 45.1766 \\
\hline $41 \ldots \ldots$ & $1 s^{2} 2 s^{2} 2 p 3 d$ & ${ }^{3} \mathrm{D}_{3}^{\circ}$ & 35.646 & 35.5992 & $1 s^{2} 2 s^{2} 2 p 3 d$ & ${ }^{3} \mathrm{D}_{3}^{\circ}$ & 45.330 & 45.3115 \\
\hline $42 \ldots \ldots$ & $1 s^{2} 2 s^{2} 2 p 3 d$ & ${ }^{3} \mathrm{P}_{2}^{\circ}$ & 35.715 & 35.6591 & $1 s^{2} 2 s^{2} 2 p 3 d$ & ${ }^{3} \mathrm{P}_{2}^{\circ}$ & 45.400 & 45.3875 \\
\hline $43 \ldots \ldots$ & $1 s^{2} 2 s^{2} 2 p 3 d$ & ${ }^{3} \mathrm{P}^{\circ}{ }_{1}$ & ...... & 35.6754 & $1 s^{2} 2 s^{2} 2 p 3 d$ & ${ }^{3} \mathrm{P}^{\circ}{ }_{1}$ & 45.430 & 45.4037 \\
\hline $44 \ldots \ldots$ & $1 s^{2} 2 s^{2} 2 p 3 d$ & ${ }^{3} \mathrm{P}_{0}^{\circ}$ & $\ldots \ldots$ & 35.6872 & $1 s^{2} 2 s^{2} 2 p 3 d$ & ${ }^{3} \mathrm{P}_{0}^{\circ}$ & $\ldots \ldots$ & 45.4177 \\
\hline $45 \ldots \ldots$ & $1 s^{2} 2 s^{2} 2 p 3 d$ & ${ }^{1} \mathrm{P}^{\circ}{ }_{1}$ & 36.075 & 36.0297 & $1 s^{2} 2 s^{2} 2 p 3 d$ & ${ }^{1} \mathrm{P}^{\circ}{ }_{1}$ & 45.820 & 45.8118 \\
\hline $46 \ldots \ldots$ & $1 s^{2} 2 s^{2} 2 p 3 d$ & ${ }^{1} \mathrm{~F}_{3}^{\circ}$ & 36.046 & 36.0519 & $1 s^{2} 2 s^{2} 2 p 3 d$ & ${ }^{1} \mathrm{~F}_{3}^{\circ}$ & 45.810 & 45.8299 \\
\hline
\end{tabular}

${ }^{a}$ NIST. 
Table 2. Target levels of Ti XVII and V XVIII, and their threshold energies (in Ryd).

\begin{tabular}{|c|c|c|c|c|c|c|c|c|}
\hline \multirow[b]{2}{*}{ Index } & \multicolumn{4}{|c|}{ Ti XVII } & \multicolumn{4}{|c|}{ V XVIII } \\
\hline & Configuration & Level & Expt. $^{a}$ & GRASP & Configuration & Level & Expt. $^{a}$ & GRASP \\
\hline $1 \ldots \ldots$ & $1 s^{2} 2 s^{2} 2 p^{2}$ & ${ }^{3} \mathrm{P}_{0}$ & 0.0000 & 0.0000 & $1 s^{2} 2 s^{2} 2 p^{2}$ & ${ }^{3} \mathrm{P}_{0}$ & 0.0000 & 0.0000 \\
\hline $2 \ldots \ldots$ & $1 s^{2} 2 s^{2} 2 p^{2}$ & ${ }^{3} \mathrm{P}_{1}$ & 0.2703 & 0.2683 & $1 s^{2} 2 s^{2} 2 p^{2}$ & ${ }^{3} \mathrm{P}_{1}$ & 0.3450 & 0.3427 \\
\hline $3 \ldots \ldots$ & $1 \mathrm{~s}^{2} 2 \mathrm{~s}^{2} 2 \mathrm{p}^{2}$ & ${ }^{3} \mathrm{P}_{2}$ & 0.5070 & 0.5078 & $1 s^{2} 2 s^{2} 2 p^{2}$ & ${ }^{3} \mathrm{P}_{2}$ & 0.6210 & 0.6216 \\
\hline $4 \ldots \ldots$. & $1 s^{2} 2 s^{2} 2 p^{2}$ & ${ }^{1} \mathrm{D}_{2}$ & 1.2817 & 1.3057 & $1 s^{2} 2 s^{2} 2 p^{2}$ & ${ }^{1} \mathrm{D}_{2}$ & 1.4663 & 1.4888 \\
\hline $5 \ldots \ldots$ & $1 \mathrm{~s}^{2} 2 \mathrm{~s}^{2} 2 \mathrm{p}^{2}$ & ${ }^{1} \mathrm{~S}_{0}$ & 2.2069 & 2.2141 & $1 s^{2} 2 s^{2} 2 p^{2}$ & ${ }^{1} \mathrm{~S}_{0}$ & 2.4500 & 2.4565 \\
\hline $6 \ldots \ldots$ & $1 s^{2} 2 s 2 p^{3}$ & ${ }^{5} \mathrm{~S}_{2}^{\circ}$ & 3.0405 & 2.9745 & $1 s^{2} 2 s 2 p^{3}$ & ${ }^{5} \mathrm{~S}_{2}^{\circ}$ & 3.3439 & 3.2770 \\
\hline $7 \ldots \ldots$. & $1 s^{2} 2 s 2 p^{3}$ & ${ }^{3} \mathrm{D}_{2}^{\circ}$ & 5.2752 & 5.3180 & $1 s^{2} 2 s 2 p^{3}$ & ${ }^{3} \mathrm{D}_{2}^{\circ}$ & 5.6850 & 5.7271 \\
\hline $8 \ldots \ldots$ & $1 s^{2} 2 s 2 p^{3}$ & ${ }^{3} \mathrm{D}^{\circ}{ }_{1}$ & 5.2863 & 5.3304 & $1 s^{2} 2 s 2 p^{3}$ & ${ }^{3} \mathrm{D}^{\circ}{ }_{1}$ & 5.6957 & 5.7402 \\
\hline $9 \ldots \ldots$. & $1 s^{2} 2 s 2 p^{3}$ & ${ }^{3} \mathrm{D}_{3}^{\circ}$ & 5.3469 & 5.3878 & $1 s^{2} 2 s 2 p^{3}$ & ${ }^{3} \mathrm{D}_{3}^{\circ}$ & 5.7860 & 5.8260 \\
\hline $10 \ldots \ldots$ & $1 s^{2} 2 s 2 p^{3}$ & ${ }^{3} \mathrm{P}_{0}^{\circ}$ & 6.1824 & 6.2364 & $1 \mathrm{~s}^{2} 2 \mathrm{~s} 2 \mathrm{p}^{3}$ & ${ }^{3} \mathrm{P}_{0}^{\circ}$ & 6.6692 & 6.7221 \\
\hline $11 \ldots \ldots$ & $1 s^{2} 2 s 2 p^{3}$ & ${ }^{3} \mathrm{P}^{\circ}{ }_{1}$ & 6.2049 & 6.2588 & $1 s^{2} 2 s 2 p^{3}$ & ${ }^{3} \mathrm{P}^{\circ}{ }_{1}$ & 6.7016 & 6.7542 \\
\hline $12 \ldots \ldots$ & $1 s^{2} 2 s 2 p^{3}$ & ${ }^{3} \mathrm{P}_{2}^{\circ}$ & 6.2584 & 6.3107 & $1 \mathrm{~s}^{2} 2 \mathrm{~s} 2 \mathrm{p}^{3}$ & ${ }^{3} \mathrm{P}_{2}^{\circ}$ & 6.7739 & 6.8256 \\
\hline $13 \ldots \ldots$ & $1 s^{2} 2 s 2 p^{3}$ & ${ }^{3} \mathrm{~S}_{1}^{\circ}$ & 7.6395 & 7.7588 & $1 s^{2} 2 s 2 p^{3}$ & ${ }^{3} \mathrm{~S}^{\circ}{ }_{1}$ & 8.1770 & 8.2966 \\
\hline $14 \ldots \ldots$ & $1 s^{2} 2 s 2 p^{3}$ & ${ }^{1} \mathrm{D}_{2}^{\circ}$ & 7.7014 & 7.8347 & $1 s^{2} 2 s 2 p^{3}$ & ${ }^{1} \mathrm{D}_{2}^{\circ}$ & 8.2781 & 8.4102 \\
\hline $15 \ldots \ldots$ & $1 s^{2} 2 s 2 p^{3}$ & ${ }^{1} \mathrm{P}^{\circ}{ }_{1}$ & 8.5970 & 8.7398 & $1 \mathrm{~s}^{2} 2 \mathrm{~s} 2 \mathrm{p}^{3}$ & ${ }^{1} \mathrm{P}^{\circ}{ }_{1}$ & 9.2441 & 9.3859 \\
\hline $16 \ldots \ldots$ & $1 s^{2} 2 p^{4}$ & ${ }^{3} \mathrm{P}_{2}$ & 11.586 & 11.7279 & $1 s^{2} 2 p^{4}$ & ${ }^{3} \mathrm{P}_{2}$ & 12.381 & 12.5239 \\
\hline $17 \ldots \ldots$ & $1 s^{2} 2 p^{4}$ & ${ }^{3} \mathrm{P}_{1}$ & 11.967 & 12.1049 & $1 s^{2} 2 p^{4}$ & ${ }^{3} \mathrm{P}_{1}$ & 12.856 & 12.9921 \\
\hline $18 \ldots \ldots$ & $1 s^{2} 2 p^{4}$ & ${ }^{3} \mathrm{P}_{0}$ & 12.026 & 12.1671 & $1 s^{2} 2 p^{4}$ & ${ }^{3} \mathrm{P}_{0}$ & 12.905 & 13.0481 \\
\hline $19 \ldots \ldots$ & $1 s^{2} 2 p^{4}$ & ${ }^{1} \mathrm{D}_{2}$ & 12.578 & 12.7659 & $1 \mathrm{~s}^{2} 2 \mathrm{p}^{4}$ & ${ }^{1} \mathrm{D}_{2}$ & 13.490 & 13.6767 \\
\hline $20 \ldots \ldots$ & $1 s^{2} 2 p^{4}$ & ${ }^{1} \mathrm{~S}_{0}$ & 14.187 & 14.4184 & $1 s^{2} 2 p^{4}$ & ${ }^{1} \mathrm{~S}_{0}$ & 15.202 & 15.4332 \\
\hline $21 \ldots \ldots$ & $1 s^{2} 2 s^{2} 2 p 3 s$ & ${ }^{3} \mathrm{P}_{0}^{\circ}$ & ....... & 46.8526 & $1 s^{2} 2 s^{2} 2 p 3 s$ & ${ }^{3} \mathrm{P}_{0}^{\circ}$ & ....... & 52.1481 \\
\hline $22 \ldots \ldots$ & $1 s^{2} 2 s^{2} 2 p 3 s$ & ${ }^{3} \mathrm{P}_{1}^{\circ}{ }_{1}$ & 46.870 & 46.9122 & $1 s^{2} 2 s^{2} 2 p 3 s$ & ${ }^{3} \mathrm{P}^{\circ}{ }_{1}$ & 52.170 & 52.2135 \\
\hline $23 \ldots \ldots$ & $1 s^{2} 2 s^{2} 2 p 3 s$ & ${ }^{3} \mathrm{P}_{2}^{\circ}$ & 47.320 & 47.3586 & $1 s^{2} 2 s^{2} 2 p 3 s$ & ${ }^{3} \mathrm{P}_{2}^{\circ}$ & 52.720 & 52.7656 \\
\hline $24 \ldots \ldots$ & $1 s^{2} 2 s^{2} 2 p 3 s$ & ${ }^{1} \mathrm{P}^{\circ}{ }_{1}$ & 47.000 & 47.5500 & $1 s^{2} 2 s^{2} 2 p 3 s$ & ${ }^{1} \mathrm{P}^{\circ}{ }_{1}$ & 52.890 & 52.9651 \\
\hline $25 \ldots \ldots$ & $1 s^{2} 2 s^{2} 2 p 3 p$ & ${ }^{3} \mathrm{D}_{1}$ & $\ldots \ldots$ & 48.1722 & $1 s^{2} 2 s^{2} 2 p 3 p$ & ${ }^{3} \mathrm{D}_{1}$ & $\ldots \ldots$ & 53.5478 \\
\hline $26 \ldots \ldots$ & $1 s^{2} 2 s^{2} 2 p 3 p$ & ${ }^{1} \mathrm{P}_{1}$ & $\ldots \ldots$. & 48.4584 & $1 s^{2} 2 s^{2} 2 p 3 p$ & ${ }^{1} \mathrm{P}_{1}$ & ....... & 53.8823 \\
\hline $27 \ldots \ldots$ & $1 s^{2} 2 s^{2} 2 p 3 p$ & ${ }^{3} \mathrm{D}_{2}$ & ....... & 48.4708 & $1 s^{2} 2 s^{2} 2 p 3 p$ & ${ }^{3} \mathrm{D}_{2}$ & ....... & 53.8979 \\
\hline $28 \ldots \ldots$ & $1 s^{2} 2 s^{2} 2 p 3 p$ & ${ }^{3} \mathrm{P}_{0}$ & $\ldots \ldots$. & 48.6474 & $1 s^{2} 2 s^{2} 2 p 3 p$ & ${ }^{3} \mathrm{P}_{0}$ & ....... & 54.0667 \\
\hline $29 \ldots \ldots$ & $1 s^{2} 2 s^{2} 2 p 3 p$ & ${ }^{3} \mathrm{P}_{1}$ & ....... & 48.8317 & $1 s^{2} 2 s^{2} 2 p 3 p$ & ${ }^{3} \mathrm{P}_{1}$ & ....... & 54.3316 \\
\hline $30 \ldots \ldots$ & $1 s^{2} 2 s^{2} 2 p 3 p$ & ${ }^{3} \mathrm{D}_{3}$ & $\ldots \ldots$ & 48.8459 & $1 s^{2} 2 s^{2} 2 p 3 p$ & ${ }^{3} \mathrm{D}_{3}$ & ....... & 54.3637 \\
\hline $31 \ldots \ldots$ & $1 s^{2} 2 s^{2} 2 p 3 p$ & ${ }^{3} S_{1}$ & ....... & 48.9724 & $1 s^{2} 2 s^{2} 2 p 3 p$ & ${ }^{3} S_{1}$ & ....... & 54.4834 \\
\hline $32 \ldots \ldots$ & $1 s^{2} 2 s^{2} 2 p 3 p$ & ${ }^{3} \mathrm{P}_{2}$ & $\ldots \ldots$. & 49.0232 & $1 s^{2} 2 s^{2} 2 p 3 p$ & ${ }^{3} \mathrm{P}_{2}$ & ....... & 54.5329 \\
\hline $33 \ldots \ldots$ & $1 s^{2} 2 s^{2} 2 p 3 p$ & ${ }^{1} D_{2}$ & $\ldots \ldots$ & 49.4567 & $1 s^{2} 2 s^{2} 2 p 3 p$ & ${ }^{1} \mathrm{D}_{2}$ & ...... & 54.9960 \\
\hline $34 \ldots \ldots$ & $1 s^{2} 2 s^{2} 2 p 3 d$ & ${ }^{3} \mathrm{~F}_{2}^{\circ}$ & 49.860 & 49.8455 & $1 \mathrm{~s}^{2} 2 \mathrm{~s}^{2} 2 \mathrm{p} 3 \mathrm{~d}$ & ${ }^{3} \mathrm{~F}_{2}^{\circ}$ & ....... & 55.3380 \\
\hline $35 \ldots \ldots$ & $1 s^{2} 2 s^{2} 2 p 3 p$ & ${ }^{1} \mathrm{~S}_{0}$ & $\ldots \ldots$ & 49.9541 & $1 s^{2} 2 s^{2} 2 p 3 p$ & ${ }^{1} \mathrm{~S}_{0}$ & $\ldots \ldots$ & 55.5161 \\
\hline $36 \ldots \ldots$ & $1 s^{2} 2 s^{2} 2 p 3 d$ & ${ }^{3} \mathrm{~F}_{3}^{\circ}$ & ....... & 50.0419 & $1 s^{2} 2 s^{2} 2 p 3 d$ & ${ }^{3} \mathrm{~F}_{3}^{\circ}$ & ....... & 55.5678 \\
\hline $37 \ldots \ldots$ & $1 s^{2} 2 s^{2} 2 p 3 d$ & ${ }^{1} \mathrm{D}_{2}^{\circ}$ & 50.130 & 50.1092 & $1 s^{2} 2 s^{2} 2 p 3 d$ & ${ }^{1} \mathrm{D}_{2}^{\circ}$ & ....... & 55.6347 \\
\hline $38 \ldots \ldots$ & $1 s^{2} 2 s^{2} 2 p 3 d$ & ${ }^{3} \mathrm{D}^{\circ}{ }_{1}$ & 50.290 & 50.2727 & $1 s^{2} 2 s^{2} 2 p 3 d$ & ${ }^{3} \mathrm{D}^{\circ}{ }_{1}$ & $\ldots \ldots$ & 55.7988 \\
\hline $39 \ldots \ldots$ & $1 \mathrm{~s}^{2} 2 \mathrm{~s}^{2} 2 \mathrm{p} 3 \mathrm{~d}$ & ${ }^{3} \mathrm{~F}^{\circ}{ }_{4}$ & ....... & 50.3724 & $1 s^{2} 2 s^{2} 2 p 3 d$ & ${ }^{3} \mathrm{~F}_{4}^{\circ}$ & $\ldots \ldots$ & 55.9829 \\
\hline $40 \ldots \ldots$ & $1 s^{2} 2 s^{2} 2 p 3 d$ & ${ }^{3} \mathrm{D}_{2}^{\circ}$ & 50.500 & 50.4702 & $1 s^{2} 2 s^{2} 2 p 3 d$ & ${ }^{3} \mathrm{D}_{2}^{\circ}$ & 56.100 & 56.0732 \\
\hline $41 \ldots \ldots$ & $1 s^{2} 2 s^{2} 2 p 3 d$ & ${ }^{3} \mathrm{D}_{3}^{\circ}$ & 50.630 & 50.6220 & $1 s^{2} 2 s^{2} 2 p 3 d$ & ${ }^{3} \mathrm{D}_{3}^{\circ}$ & 56.260 & 56.2403 \\
\hline $42 \ldots \ldots$ & $1 s^{2} 2 s^{2} 2 p 3 d$ & ${ }^{3} \mathrm{P}_{2}^{\circ}$ & 50.730 & 50.7071 & $1 \mathrm{~s}^{2} 2 \mathrm{~s}^{2} 2 \mathrm{p} 3 \mathrm{~d}$ & ${ }^{3} \mathrm{P}_{2}^{\circ}$ & 56.380 & 56.3353 \\
\hline $43 \ldots \ldots$ & $1 s^{2} 2 s^{2} 2 p 3 d$ & ${ }^{3} \mathrm{P}^{\circ}{ }_{1}$ & $\ldots \ldots$. & 50.7224 & $1 \mathrm{~s}^{2} 2 \mathrm{~s}^{2} 2 \mathrm{p} 3 \mathrm{~d}$ & ${ }^{3} \mathrm{P}^{\circ}{ }_{1}$ & 56.450 & 56.3488 \\
\hline $44 \ldots \ldots$ & $1 s^{2} 2 s^{2} 2 p 3 d$ & ${ }^{3} \mathrm{P}_{0}^{\circ}$ & ....... & 50.7374 & $1 s^{2} 2 s^{2} 2 p 3 d$ & ${ }^{3} \mathrm{P}_{0}^{\circ}$ & $\ldots \ldots$ & 56.3646 \\
\hline $45 \ldots \ldots$ & $1 s^{2} 2 s^{2} 2 p 3 d$ & ${ }^{1} \mathrm{P}^{\circ}{ }_{1}$ & 51.140 & 51.1544 & $1 s^{2} 2 s^{2} 2 p 3 d$ & ${ }^{1} \mathrm{P}^{\circ}{ }_{1}$ & $\ldots \ldots$ & 56.8048 \\
\hline $46 \ldots \ldots$ & $1 s^{2} 2 s^{2} 2 p 3 d$ & ${ }^{1} \mathrm{~F}_{3}^{\circ}$ & 51.150 & 51.1723 & $1 s^{2} 2 s^{2} 2 p 3 d$ & ${ }^{1} \mathrm{~F}_{3}^{\circ}$ & 56.800 & 56.8221 \\
\hline
\end{tabular}


Table 3. Target levels of Cr XIX and Mn XX, and their threshold energies (in Ryd).

\begin{tabular}{|c|c|c|c|c|c|c|c|c|}
\hline \multirow[b]{2}{*}{ Index } & \multicolumn{4}{|c|}{ Cr XIX } & \multicolumn{4}{|c|}{$\operatorname{Mn} X X$} \\
\hline & Configuration & Level & Expt. $^{a}$ & GRASP & Configuration & Level & Expt. $^{a}$ & GRASP \\
\hline $1 \ldots \ldots$ & $1 s^{2} 2 s^{2} 2 p^{2}$ & ${ }^{3} \mathrm{P}_{0}$ & 0.0000 & 0.0000 & $1 \mathrm{~s}^{2} 2 \mathrm{~s}^{2} 2 \mathrm{p}^{2}$ & ${ }^{3} \mathrm{P}_{0}$ & 0.0000 & 0.0000 \\
\hline $2 \ldots \ldots$ & $1 s^{2} 2 s^{2} 2 p^{2}$ & ${ }^{3} \mathrm{P}_{1}$ & 0.4357 & 0.4329 & $1 \mathrm{~s}^{2} 2 \mathrm{~s}^{2} 2 \mathrm{p}^{2}$ & ${ }^{3} \mathrm{P}_{1}$ & 0.5420 & 0.5410 \\
\hline $3 \ldots \ldots$ & $1 s^{2} 2 s^{2} 2 p^{2}$ & ${ }^{3} \mathrm{P}_{2}$ & 0.7514 & 0.7525 & $1 s^{2} 2 s^{2} 2 p^{2}$ & ${ }^{3} \mathrm{P}_{2}$ & 0.8980 & 0.9020 \\
\hline $4 \ldots \ldots$ & $1 \mathrm{~s}^{2} 2 \mathrm{~s}^{2} 2 \mathrm{p}^{2}$ & ${ }^{1} \mathrm{D}_{2}$ & 1.6822 & 1.7034 & $1 \mathrm{~s}^{2} 2 \mathrm{~s}^{2} 2 \mathrm{p}^{2}$ & ${ }^{1} D_{2}$ & 1.9342 & 1.9540 \\
\hline $5 \ldots \ldots$ & $1 s^{2} 2 s^{2} 2 p^{2}$ & ${ }^{1} \mathrm{~S}_{0}$ & 2.7220 & 2.7311 & $1 s^{2} 2 s^{2} 2 p^{2}$ & ${ }^{1} \mathrm{~S}_{0}$ & 3.0352 & 3.0422 \\
\hline $6 \ldots \ldots$ & $1 s^{2} 2 s 2 p^{3}$ & ${ }^{5} \mathrm{~S}_{2}^{\circ}$ & 3.6748 & 3.6090 & $1 \mathrm{~s}^{2} 2 \mathrm{~s} 2 \mathrm{p}^{3}$ & ${ }^{5} \mathrm{~S}_{2}^{\circ}$ & 4.0374 & 3.9730 \\
\hline $7 \ldots \ldots$ & $1 s^{2} 2 s 2 p^{3}$ & ${ }^{3} \mathrm{D}_{2}^{\circ}$ & 6.1203 & 6.1628 & $1 s^{2} 2 s 2 p^{3}$ & ${ }^{3} \mathrm{D}_{2}^{\circ}$ & 6.5858 & 6.6282 \\
\hline $8 \ldots \ldots$ & $1 s^{2} 2 s 2 p^{3}$ & ${ }^{3} \mathrm{D}^{\circ}{ }_{1}$ & 6.1307 & 6.1747 & $1 s^{2} 2 s 2 p^{3}$ & ${ }^{3} \mathrm{D}^{\circ}{ }_{1}$ & 6.5892 & 6.6358 \\
\hline $9 \ldots \ldots$ & $1 s^{2} 2 s 2 p^{3}$ & ${ }^{3} \mathrm{D}_{3}^{\circ}$ & 6.2588 & 6.2985 & $1 \mathrm{~s}^{2} 2 \mathrm{~s} 2 \mathrm{p}^{3}$ & ${ }^{3} \mathrm{D}_{3}^{\circ}$ & 6.7701 & 6.8092 \\
\hline $10 \ldots \ldots$ & $1 \mathrm{~s}^{2} 2 \mathrm{~s} 2 \mathrm{p}^{3}$ & ${ }^{3} \mathrm{P}_{0}^{\circ}$ & 7.1913 & 7.2424 & $1 s^{2} 2 s 2 p^{3}$ & ${ }^{3} \mathrm{P}_{0}^{\circ}$ & 7.7488 & 7.8016 \\
\hline $11 \ldots \ldots$ & $1 s^{2} 2 s^{2} p^{3}$ & ${ }^{3} \mathrm{P}_{1}^{\circ}$ & 7.2366 & 7.2871 & $1 s^{2} 2 s 2 p^{3}$ & ${ }^{3} \mathrm{P}_{1}^{\circ}$ & 7.8080 & 7.8617 \\
\hline $12 \ldots \ldots$ & $1 s^{2} 2 s 2 p^{3}$ & ${ }^{3} \mathrm{P}_{2}^{\circ}$ & 7.3334 & 7.3826 & $1 s^{2} 2 s 2 p^{3}$ & ${ }^{3} \mathrm{P}_{2}^{\circ}$ & 7.9333 & 7.9863 \\
\hline $13 \ldots \ldots$ & $1 s^{2} 2 s 2 p^{3}$ & ${ }^{3} \mathrm{~S}_{1}^{\circ}$ & 8.7470 & 8.8644 & $1 s^{2} 2 s 2 p^{3}$ & ${ }^{3} S^{\circ}{ }_{1}$ & 9.3451 & 9.4660 \\
\hline $14 \ldots \ldots$ & $1 s^{2} 2 s 2 p^{3}$ & ${ }^{1} \mathrm{D}_{2}^{\circ}$ & 8.8959 & 9.0259 & $1 s^{2} 2 s 2 p^{3}$ & ${ }^{1} \mathrm{D}_{2}^{\circ}$ & 9.5581 & 9.6872 \\
\hline $15 \ldots \ldots$ & $1 s^{2} 2 s 2 p^{3}$ & ${ }^{1} \mathrm{P}^{\circ}{ }_{1}$ & 9.9388 & 10.0787 & $1 s^{2} 2 s 2 p^{3}$ & ${ }^{1} \mathrm{P}_{1}^{\circ}$ & 10.685 & 10.8245 \\
\hline $16 \ldots \ldots$ & $1 s^{2} 2 p^{4}$ & ${ }^{3} \mathrm{P}_{2}$ & 13.215 & 13.3569 & $1 s^{2} 2 p^{4}$ & ${ }^{3} \mathrm{P}_{2}$ & 14.086 & 14.2310 \\
\hline $17 \ldots \ldots$ & $1 s^{2} 2 p^{4}$ & ${ }^{3} \mathrm{P}_{1}$ & 13.800 & 13.9329 & $1 s^{2} 2 p^{4}$ & ${ }^{3} \mathrm{P}_{1}$ & 14.796 & 14.9331 \\
\hline $18 \ldots \ldots$ & $1 s^{2} 2 p^{4}$ & ${ }^{3} \mathrm{P}_{0}$ & 13.833 & 13.9737 & $1 \mathrm{~s}^{2} 2 \mathrm{p}^{4}$ & ${ }^{3} \mathrm{P}_{0}$ & 14.798 & 14.9467 \\
\hline $19 \ldots \ldots$ & $1 s^{2} 2 p^{4}$ & ${ }^{1} \mathrm{D}_{2}$ & 14.455 & 14.6399 & $1 \mathrm{~s}^{2} 2 \mathrm{p}^{4}$ & ${ }^{1} \mathrm{D}_{2}$ & 15.476 & 15.6618 \\
\hline $20 \ldots \ldots$ & $1 \mathrm{~s}^{2} 2 \mathrm{p}^{4}$ & ${ }^{1} \mathrm{~S}_{0}$ & 16.286 & 16.5100 & $1 \mathrm{~s}^{2} 2 \mathrm{p}^{4}$ & ${ }^{1} \mathrm{~S}_{0}$ & 17.432 & 17.6580 \\
\hline $21 \ldots \ldots$ & $1 s^{2} 2 s^{2} 2 p 3 s$ & ${ }^{3} \mathrm{P}_{0}^{\circ}$ & ....... & 57.7323 & $1 s^{2} 2 s^{2} 2 p 3 s$ & ${ }^{3} \mathrm{P}_{0}^{\circ}$ & ....... & 63.6071 \\
\hline $22 \ldots \ldots$ & $1 s^{2} 2 s^{2} 2 p 3 s$ & ${ }^{3} \mathrm{P}_{1}^{\circ}$ & $\ldots \ldots$ & 57.8035 & $1 s^{2} 2 s^{2} 2 p 3 s$ & ${ }^{3} \mathrm{P}_{1}^{\circ}$ & $\ldots \ldots$ & 63.6839 \\
\hline $23 \ldots \ldots$ & $1 s^{2} 2 s^{2} 2 p 3 s$ & ${ }^{3} \mathrm{P}_{2}^{\circ}$ & ....... & 58.4790 & $1 s^{2} 2 s^{2} 2 p 3 s$ & ${ }^{3} \mathrm{P}^{\circ}{ }_{2}$ & ....... & 64.5025 \\
\hline $24 \ldots \ldots$ & $1 s^{2} 2 s^{2} 2 p 3 s$ & ${ }^{1} \mathrm{P}^{\circ}{ }_{1}$ & ....... & 58.6867 & $1 s^{2} 2 s^{2} 2 p 3 s$ & ${ }^{1} \mathrm{P}^{\circ}{ }_{1}$ & ....... & 64.7184 \\
\hline $25 \ldots \ldots$ & $1 s^{2} 2 s^{2} 2 p 3 p$ & ${ }^{3} D_{1}$ & ....... & 59.2125 & $1 s^{2} 2 s^{2} 2 p 3 p$ & ${ }^{3} \mathrm{D}_{1}$ & ....... & 65.1680 \\
\hline $26 \ldots \ldots$ & $1 s^{2} 2 s^{2} 2 p 3 p$ & ${ }^{1} \mathrm{P}_{1}$ & ....... & 59.5998 & $1 s^{2} 2 s^{2} 2 p 3 p$ & ${ }^{1} \mathrm{P}_{1}$ & $\ldots .$. & 65.6133 \\
\hline $27 \ldots \ldots$ & $1 s^{2} 2 s^{2} 2 p 3 p$ & ${ }^{3} \mathrm{D}_{2}$ & ....... & 59.6202 & $1 s^{2} 2 s^{2} 2 p 3 p$ & ${ }^{3} \mathrm{D}_{2}$ & $\ldots$. & 65.6400 \\
\hline $28 \ldots \ldots$ & $1 s^{2} 2 s^{2} 2 p 3 p$ & ${ }^{3} \mathrm{P}_{0}$ & ....... & 59.7770 & $1 s^{2} 2 s^{2} 2 p 3 p$ & ${ }^{3} \mathrm{P}_{0}$ & ....... & 65.7798 \\
\hline $29 \ldots \ldots$ & $1 s^{2} 2 s^{2} 2 p 3 p$ & ${ }^{3} \mathrm{P}_{1}$ & ....... & 60.1408 & $1 s^{2} 2 s^{2} 2 p 3 p$ & ${ }^{3} \mathrm{P}_{1}$ & $\ldots \ldots$ & 66.2625 \\
\hline $30 \ldots \ldots$ & $1 s^{2} 2 s^{2} 2 p 3 p$ & ${ }^{3} \mathrm{D}_{3}$ & ....... & 60.1932 & $1 s^{2} 2 s^{2} 2 p 3 p$ & ${ }^{3} \mathrm{D}_{3}$ & ....... & 66.3386 \\
\hline $31 \ldots \ldots$ & $1 s^{2} 2 s^{2} 2 p 3 p$ & ${ }^{3} S_{1}$ & ....... & 60.3047 & $1 s^{2} 2 s^{2} 2 p 3 p$ & ${ }^{3} S_{1}$ & ....... & 66.4407 \\
\hline $32 \ldots \ldots$ & $1 s^{2} 2 s^{2} 2 p 3 p$ & ${ }^{3} \mathrm{P}_{2}$ & ....... & 60.3497 & $1 s^{2} 2 s^{2} 2 p 3 p$ & ${ }^{3} \mathrm{P}_{2}$ & $\ldots$. & 66.4770 \\
\hline $33 \ldots \ldots$ & $1 s^{2} 2 s^{2} 2 p 3 p$ & ${ }^{1} \mathrm{D}_{2}$ & ....... & 60.8463 & $1 s^{2} 2 s^{2} 2 p 3 p$ & ${ }^{1} D_{2}$ & ....... & 67.0120 \\
\hline $34 \ldots \ldots$ & $1 \mathrm{~s}^{2} 2 \mathrm{~s}^{2} 2 \mathrm{p} 3 \mathrm{~d}$ & ${ }^{3} \mathrm{~F}_{2}^{\circ}$ & ....... & 61.1247 & $1 \mathrm{~s}^{2} 2 \mathrm{~s}^{2} 2 \mathrm{p} 3 \mathrm{~d}$ & ${ }^{3} \mathrm{~F}_{2}^{\circ}$ & ....... & 67.2078 \\
\hline $35 \ldots \ldots$ & $1 s^{2} 2 s^{2} 2 p 3 p$ & ${ }^{1} \mathrm{~S}_{0}$ & ....... & 61.3854 & $1 s^{2} 2 s^{2} 2 p 3 d$ & ${ }^{3} \mathrm{~F}_{3}^{\circ}$ & ....... & 67.5120 \\
\hline $36 \ldots \ldots$ & $1 s^{2} 2 s^{2} 2 p 3 d$ & ${ }^{3} \mathrm{~F}_{3}^{\circ}$ & ....... & 61.3904 & $1 s^{2} 2 s^{2} 2 p 3 p$ & ${ }^{1} \mathrm{~S}_{0}$ & ....... & 67.5645 \\
\hline $37 \ldots \ldots$ & $1 s^{2} 2 s^{2} 2 p 3 d$ & ${ }^{1} \mathrm{D}_{2}^{\circ}$ & $\ldots \ldots$. & 61.4547 & $1 s^{2} 2 s^{2} 2 p 3 d$ & ${ }^{1} \mathrm{D}_{2}^{\circ}$ & ....... & 67.5718 \\
\hline $38 \ldots \ldots$ & $1 s^{2} 2 s^{2} 2 p 3 d$ & ${ }^{3} \mathrm{D}^{\circ}{ }_{1}$ & ....... & 61.6191 & $1 s^{2} 2 s^{2} 2 p 3 d$ & ${ }^{3} \mathrm{D}^{\circ}{ }_{1}$ & ....... & 67.7359 \\
\hline $39 \ldots \ldots$ & $1 s^{2} 2 s^{2} 2 p 3 d$ & ${ }^{3} \mathrm{~F}_{4}^{\circ}$ & ....... & 61.9066 & $1 s^{2} 2 s^{2} 2 p 3 d$ & ${ }^{3} \mathrm{~F}^{\circ}{ }_{4}$ & $\ldots \ldots$ & 68.1478 \\
\hline $40 \ldots \ldots$ & $1 s^{2} 2 s^{2} 2 p 3 d$ & ${ }^{3} \mathrm{D}_{2}^{\circ}$ & ....... & 61.9896 & $1 \mathrm{~s}^{2} 2 \mathrm{~s}^{2} 2 \mathrm{p} 3 \mathrm{~d}$ & ${ }^{3} \mathrm{D}_{2}^{\circ}$ & $\ldots \ldots$ & 68.2234 \\
\hline $41 \ldots \ldots$ & $1 s^{2} 2 s^{2} 2 p 3 d$ & ${ }^{3} \mathrm{D}_{3}^{\circ}$ & ....... & 62.1706 & $1 s^{2} 2 s^{2} 2 p 3 d$ & ${ }^{3} \mathrm{D}_{3}^{\circ}$ & ....... & 68.4171 \\
\hline $42 \ldots \ldots$ & $1 s^{2} 2 s^{2} 2 p 3 d$ & ${ }^{3} \mathrm{P}_{2}^{\circ}$ & ....... & 62.2760 & $1 s^{2} 2 s^{2} 2 p 3 d$ & ${ }^{3} \mathrm{P}_{2}^{\circ}$ & ....... & 68.5336 \\
\hline $43 \ldots \ldots$ & $1 s^{2} 2 s^{2} 2 p 3 d$ & ${ }^{3} \mathrm{P}^{\circ}{ }_{1}$ & ....... & 62.2865 & $1 s^{2} 2 s^{2} 2 p 3 d$ & ${ }^{3} \mathrm{P}_{1}^{\circ}$ & ....... & 68.5390 \\
\hline $44 \ldots \ldots$ & $1 s^{2} 2 s^{2} 2 p 3 d$ & ${ }^{3} \mathrm{P}_{0}^{\circ}$ & ....... & 62.3028 & $1 s^{2} 2 s^{2} 2 p 3 d$ & ${ }^{3} \mathrm{P}_{0}^{\circ}$ & ....... & 68.5544 \\
\hline $45 \ldots \ldots$ & $1 s^{2} 2 s^{2} 2 p 3 d$ & ${ }^{1} \mathrm{P}^{\circ}{ }_{1}$ & ....... & 62.7629 & $1 \mathrm{~s}^{2} 2 \mathrm{~s}^{2} 2 \mathrm{p} 3 \mathrm{~d}$ & ${ }^{1} \mathrm{P}_{1}^{\circ}$ & ....... & 69.0318 \\
\hline $46 \ldots \ldots$ & $1 s^{2} 2 s^{2} 2 p 3 d$ & ${ }^{1} \mathrm{~F}_{3}^{\circ}$ & $\ldots \ldots$. & 62.7836 & $1 s^{2} 2 s^{2} 2 p 3 d$ & ${ }^{1} \mathrm{~F}_{3}^{\circ}$ & ....... & 69.0611 \\
\hline
\end{tabular}

${ }^{a}$ NIST. 
Table 10. Comparison between our present GRASP (first rows) oscillator strengths with the HFR (second rows) results of Fawcett (1987) for a few transitions of C-like ions. $\left(a \pm b \equiv a \times 10^{ \pm b}\right)$.

\begin{tabular}{|c|c|c|c|c|c|c|c|c|}
\hline$i$ & $j$ & Transition & K XIV & Sc XVI & Ti XVII & V XVIII & Cr XIX & $\operatorname{Mn} X X$ \\
\hline \multirow[t]{2}{*}{1} & \multirow[t]{2}{*}{22} & \multirow[t]{2}{*}{${ }^{3} \mathrm{P}_{0}-{ }^{3} \mathrm{P}^{\circ}{ }_{1}$} & $5.995-2$ & $5.813-2$ & $5.748-2$ & $5.697-2$ & $5.655-2$ & 5.619-2 \\
\hline & & & $6.600-2$ & $6.400-2$ & $6.400-2$ & $6.300-2$ & $6.300-2$ & $6.300-2$ \\
\hline \multirow[t]{2}{*}{1} & \multirow[t]{2}{*}{24} & \multirow{2}{*}{${ }^{3} \mathrm{P}_{0}-{ }^{1} \mathrm{P}^{\circ}{ }_{1}$} & $2.042-3$ & $2.044-3$ & $1.941-3$ & $1.789-3$ & $1.605-3$ & $1.404-3$ \\
\hline & & & $2.000-3$ & $2.000-3$ & $2.000-3$ & $2.000-3$ & $2.000-3$ & $2.000-2$ \\
\hline \multirow[t]{2}{*}{1} & \multirow[t]{2}{*}{39} & \multirow[t]{2}{*}{${ }^{3} \mathrm{P}_{0}-{ }^{3} \mathrm{D}^{\circ}{ }_{1}$} & $1.235-0$ & $1.262-0$ & $1.274-0$ & $1.285-0$ & $1.295-0$ & $1.305-0$ \\
\hline & & & $1.320-0$ & $1.340-0$ & $1.349-0$ & $1.359-0$ & $1.368-0$ & $1.377-0$ \\
\hline \multirow[t]{2}{*}{2} & \multirow[t]{2}{*}{37} & \multirow{2}{*}{${ }^{3} \mathrm{P}_{1}-{ }^{1} \mathrm{D}_{2}^{\circ}$} & $1.507-1$ & $2.593-1$ & $3.113-1$ & $3.567-1$ & 3.944-1 & $4.250-1$ \\
\hline & & & $1.600-1$ & $2.720-1$ & $3.253-1$ & $3.713-1$ & $3.790-1$ & $3.487-1$ \\
\hline \multirow[t]{2}{*}{2} & \multirow[t]{2}{*}{39} & \multirow[t]{2}{*}{${ }^{3} \mathrm{P}_{1}-{ }^{3} \mathrm{D}^{\circ}{ }_{1}$} & $1.039-1$ & $9.406-2$ & $9.002-2$ & $8.645-2$ & $8.327-2$ & $8.045-2$ \\
\hline & & & $1.077-1$ & $9.500-2$ & $9.100-2$ & $8.730-2$ & $8.430-2$ & $8.130-2$ \\
\hline \multirow[t]{2}{*}{2} & \multirow[t]{2}{*}{40} & \multirow{2}{*}{${ }^{3} \mathrm{P}_{1}-{ }^{3} \mathrm{D}_{2}^{\circ}$} & $6.162-1$ & $5.015-1$ & $4.480-1$ & $4.019-1$ & $3.643-1$ & $3.347-1$ \\
\hline & & & $6.400-1$ & $5.200-1$ & $4.647-1$ & $4.177-1$ & $4.090-1$ & 4.397-1 \\
\hline \multirow[t]{2}{*}{2} & \multirow[t]{2}{*}{42} & ${ }^{3} \mathrm{P}_{1}-{ }^{3} \mathrm{P}_{2}^{\circ}$ & $6.010-2$ & $7.564-2$ & $8.093-2$ & $8.484-2$ & $8.756-2$ & $8.927-2$ \\
\hline & & & $7.700-2$ & $8.970-2$ & $9.400-2$ & $9.700-2$ & $9.900-2$ & $1.000-1$ \\
\hline 2 & 43 & ${ }^{3} \mathrm{P}_{1}-{ }^{3} \mathrm{P}_{1}^{\circ}$ & 2.081-1 & $2.226-1$ & $2.290-1$ & $2.352-1$ & $2.414-1$ & $2.482-1$ \\
\hline & & & $2.280-1$ & $2.400-1$ & $2.457-1$ & $2.510-1$ & $2.563-1$ & $2.617-1$ \\
\hline 2 & 44 & ${ }^{3} \mathrm{P}_{1}-{ }^{3} \mathrm{P}_{0}^{\circ}$ & $1.035-1$ & $1.051-1$ & $1.059-1$ & $1.066-1$ & $1.072-1$ & $1.072-1$ \\
\hline & & & $1.100-1$ & $1.110-1$ & $1.120-1$ & $1.130-1$ & $1.133-1$ & $1.140-1$ \\
\hline 2 & 45 & ${ }^{3} \mathrm{P}_{1}-{ }^{1} \mathrm{P}^{\circ}{ }_{1}$ & $5.607-3$ & $7.545-4$ & $6.570-3$ & $1.004-2$ & $1.421-2$ & $2.039-2$ \\
\hline & & & $7.700-3$ & $6.700-3$ & $9.000-3$ & $8.000-3$ & $8.700-3$ & $1.070-2$ \\
\hline 3 & 22 & ${ }^{3} \mathrm{P}_{2}-{ }^{3} \mathrm{P}_{1}^{\circ}$ & $1.695-2$ & $1.760-2$ & $1.808-2$ & $1.863-2$ & $1.921-2$ & $1.979-2$ \\
\hline & & & $1.840-2$ & $1.960-2$ & $1.960-2$ & $2.020-2$ & $2.080-2$ & $2.140-2$ \\
\hline 3 & 23 & ${ }^{3} \mathrm{P}_{2}-{ }^{3} \mathrm{P}_{2}^{\circ}$ & $4.530-2$ & $4.247-2$ & $4.088-2$ & $3.918-2$ & $3.737-2$ & $3.550-2$ \\
\hline & & & $4.940-2$ & $4.600-2$ & $4.420-2$ & $4.220-2$ & $4.020-2$ & $3.820-2$ \\
\hline 3 & 35 & ${ }^{3} \mathrm{P}_{2}-{ }^{3} \mathrm{~F}_{2}^{\circ}$ & $1.136-2$ & $1.751-2$ & $2.107-2$ & $2.482-2$ & $2.866-2$ & $3.244-2$ \\
\hline & & & $1.060-2$ & $1.700-2$ & $2.080-2$ & $2.480-2$ & $2.880-2$ & $3.260-2$ \\
\hline 3 & 36 & ${ }^{3} \mathrm{P}_{2}-{ }^{3} \mathrm{~F}_{3}^{\circ}$ & $5.376-2$ & $8.743-2$ & $1.078-1$ & $1.300-1$ & $1.536-1$ & $1.781-1$ \\
\hline & & & $5.120-2$ & $8.540-2$ & $1.062-1$ & $1.292-1$ & $1.536-1$ & $1.788-1$ \\
\hline 3 & 37 & ${ }^{3} \mathrm{P}_{2}-{ }^{1} \mathrm{D}_{2}^{\circ}$ & $1.190-2$ & $1.988-2$ & $2.387-2$ & $2.757-2$ & $3.086-2$ & $3.367-2$ \\
\hline & & & $1.720-2$ & $2.600-2$ & $3.020-2$ & $3.400-2$ & $2.300-2$ & $3.120-2$ \\
\hline 3 & 41 & ${ }^{3} \mathrm{P}_{2}-{ }^{3} \mathrm{D}_{3}^{\circ}$ & $7.378-1$ & $7.170-1$ & $7.020-1$ & $6.845-1$ & $6.650-1$ & $6.443-1$ \\
\hline & & & $7.902-1$ & $7.642-1$ & $7.466-1$ & $7.264-1$ & $7.040-1$ & $6.808-1$ \\
\hline 3 & 42 & ${ }^{3} \mathrm{P}_{2}-{ }^{3} \mathrm{P}_{2}^{\circ}$ & $3.398-1$ & $3.213-1$ & $3.082-1$ & $2.927-1$ & $2.753-1$ & $2.565-1$ \\
\hline & & & $3.564-1$ & $3.328-1$ & $3.174-1$ & $2.998-1$ & 2.804-1 & $2.600-1$ \\
\hline 3 & 43 & ${ }^{3} \mathrm{P}_{2}-{ }^{3} \mathrm{P}^{\circ}{ }_{1}$ & $8.213-2$ & $7.940-2$ & $7.726-2$ & $7.453-2$ & 7.114-2 & $6.678-2$ \\
\hline & & & $8.580-2$ & $8.440-2$ & $7.980-2$ & $7.680-2$ & $7.370-2$ & $6.920-2$ \\
\hline 3 & 46 & ${ }^{3} \mathrm{P}_{2}-{ }^{1} \mathrm{~F}_{3}^{\circ}$ & $4.921-3$ & $1.299-2$ & $1.943-2$ & $2.760-2$ & $3.730-2$ & 4.809-2 \\
\hline & & & $6.400-3$ & $1.600-2$ & $2.360-2$ & $3.320-2$ & $4.420-2$ & $5.640-2$ \\
\hline 4 & 24 & ${ }^{1} \mathrm{D}_{2}-{ }^{1} \mathrm{P}^{\circ}{ }_{1}$ & $4.696-2$ & $4.428-2$ & $4.305-2$ & $4.185-2$ & $4.066-2$ & $3.947-2$ \\
\hline & & & $5.440-2$ & $5.040-2$ & $4.860-2$ & $4.700-2$ & $4.520-2$ & $4.360-2$ \\
\hline 4 & 35 & ${ }^{1} \mathrm{D}_{2}-{ }^{3} \mathrm{~F}_{2}^{\circ}$ & $3.957-2$ & $3.449-2$ & $3.153-2$ & $2.839-2$ & $2.517-2$ & $2.198-2$ \\
\hline & & & $5.280-2$ & $3.060-2$ & $2.860-2$ & $2.600-2$ & $2.320-2$ & $2.040-2$ \\
\hline 4 & 37 & ${ }^{1} \mathrm{D}_{2}-{ }^{1} \mathrm{D}_{2}^{\circ}$ & $1.170-1$ & $8.670-2$ & $7.182-2$ & $5.856-2$ & $4.729-2$ & $3.798-2$ \\
\hline & & & $1.356-1$ & $9.600-2$ & $7.840-2$ & $6.300-2$ & $5.020-2$ & $7.540-2$ \\
\hline 4 & 42 & ${ }^{1} \mathrm{D}_{2}-{ }^{3} \mathrm{P}_{2}^{\circ}$ & $2.728-2$ & $4.809-2$ & $6.188-2$ & $7.782-2$ & $9.554-2$ & $1.145-1$ \\
\hline & & & $3.120-2$ & $5.480-2$ & $7.020-2$ & $8.740-2$ & $1.064-1$ & $1.262-1$ \\
\hline 4 & 45 & ${ }^{1} \mathrm{D}_{2}-{ }^{1} \mathrm{P}^{\circ}{ }_{1}$ & $1.263-2$ & $9.475-3$ & $1.235-2$ & $1.292-2$ & $1.332-2$ & $1.330-2$ \\
\hline & & & $7.400-3$ & $8.200-3$ & $8.600-3$ & $7.400-3$ & $8.800-3$ & $9.400-3$ \\
\hline
\end{tabular}


Table 10. continued.

\begin{tabular}{|c|c|c|c|c|c|c|c|c|}
\hline$i$ & $j$ & Transition & K XIV & Sc XVI & Ti XVII & V XVIII & Cr XIX & Mn XX \\
\hline \multirow[t]{2}{*}{4} & \multirow[t]{2}{*}{46} & \multirow[t]{2}{*}{${ }^{1} \mathrm{D}_{2}-{ }^{1} \mathrm{~F}_{3}^{\circ}$} & $1.032-0$ & $1.033-0$ & $1.028-0$ & $1.020-0$ & 1.009-0 & 9.956-1 \\
\hline & & & $1.072-0$ & $1.068-0$ & $1.060-0$ & $1.050-0$ & $1.036-0$ & $1.020-0$ \\
\hline \multirow[t]{2}{*}{5} & \multirow[t]{2}{*}{22} & \multirow[t]{2}{*}{${ }^{1} \mathrm{~S}_{0}-{ }^{3} \mathrm{P}^{\circ}{ }_{1}$} & $3.358-3$ & $3.595-3$ & $3.560-3$ & $3.443-3$ & $3.263-3$ & $3.041-3$ \\
\hline & & & $4.000-3$ & $4.000-3$ & $4.000-3$ & $4.000-3$ & $4.000-3$ & $4.000-3$ \\
\hline \multirow[t]{2}{*}{5} & \multirow[t]{2}{*}{24} & \multirow[t]{2}{*}{${ }^{1} \mathrm{~S}_{0}-{ }^{1} \mathrm{P}^{\circ}{ }_{1}$} & $7.402-2$ & $7.097-2$ & $6.978-2$ & $6.876-2$ & $6.788-2$ & $6.712-2$ \\
\hline & & & $7.800-2$ & $7.500-2$ & $7.300-2$ & $7.200-2$ & $7.100-2$ & $7.000-2$ \\
\hline \multirow[t]{2}{*}{5} & \multirow[t]{2}{*}{39} & \multirow[t]{2}{*}{${ }^{1} \mathrm{~S}_{0}-{ }^{3} \mathrm{D}^{\circ}{ }_{1}$} & $1.092-2$ & $1.320-2$ & $1.384-2$ & $1.410-2$ & $1.402-2$ & $1.362-2$ \\
\hline & & & $1.300-2$ & $1.500-2$ & $1.500-2$ & $1.500-2$ & $1.500-2$ & $1.500-2$ \\
\hline \multirow[t]{2}{*}{5} & \multirow[t]{2}{*}{43} & \multirow[t]{2}{*}{${ }^{1} \mathrm{~S}_{0}-{ }^{3} \mathrm{P}^{\circ}{ }_{1}$} & $2.246-3$ & $3.195-3$ & $3.803-3$ & $4.507-3$ & $5.294-3$ & $6.115-3$ \\
\hline & & & $2.000-3$ & $3.000-3$ & $3.000-3$ & $4.000-3$ & $4.000-3$ & $5.000-3$ \\
\hline \multirow[t]{2}{*}{5} & \multirow[t]{2}{*}{45} & \multirow[t]{2}{*}{${ }^{1} \mathrm{~S}_{0}-{ }^{1} \mathrm{P}^{\circ}{ }_{1}$} & $1.337-0$ & $1.174-0$ & $1.314-0$ & $1.281-0$ & $1.198-0$ & $1.004-0$ \\
\hline & & & $1.394-0$ & $1.387-0$ & $1.368-0$ & $1.334-0$ & $1.365-0$ & $1.344-0$ \\
\hline
\end{tabular}

significantly larger $(\geq 0.10)$, the agreement between the two forms is within $20 \%$, and the only exception is the 33-46 (2p3p ${ }^{1} \mathrm{D}_{2}-2 \mathrm{p} 3 \mathrm{~d}^{1} \mathrm{~F}_{3}^{\circ}$ ) transition in K XIV, for which the length form is higher by a factor of two. For transitions with $f$-values $\geq 0.01$, the two forms agree within a factor of two for all transitions in all ions. In fact, for a majority of weak transitions the two forms agree to better than $40 \%$. This is highly satisfactory.

The measurements of $A$-values, in the form of lifetimes, can provide a good check on the theoretical results, and hence can help to improve the accuracy. However, to the best of our knowledge there are no experimental values available in the literature for the ions considered here. Some experiments have reported the lifetimes for the $2 \mathrm{~s}^{2} 2 \mathrm{p}^{2}{ }^{1} \mathrm{~S}_{0},{ }^{1} \mathrm{D}_{2}$ and $2 \mathrm{~s} 2 \mathrm{p}^{3}{ }^{5} \mathrm{~S}_{2}^{\circ}$ levels in other C-like ions, (see, for example, Trabert 2002 and references therein). We hope experiments in the future will be performed for other C-like ions also, which will help us in improving upon the accuracy of the calculated atomic data.

\section{Conclusions}

In this paper we have reported values of oscillator strengths and radiative rates for transitions among the $\left(1 \mathrm{~s}^{2}\right) 2 \mathrm{~s}^{2} 2 \mathrm{p}^{2}, 2 \mathrm{~s} 2 \mathrm{p}^{3}$, $2 p^{4}, 2 s^{2} 2 p 3 s, 2 s^{2} 2 p 3 p$ and $2 s^{2} 2 p 3 d$ configurations of C-like K XIV, Sc XVI, Ti XVII, V XVIII, Cr XIX and Mn XX. Relativistic effects as well as elaborate $\mathrm{CI}$ have been included in the calculations. The results have been compared with the earlier available values obtained by Fawcett (1987) from the HFR code of Cowan (1981).

The energy levels agree to better than $3 \%$ with the experimental compilations, but the levels ordering are slightly different in a few instances. Furthermore, experimental energies for all the levels are not available, and the deficiency is greater for the higher levels, in particular. This may result in an incorrect order of some of the levels, leaving scope for future amendments.

The oscillator strengths for transitions among the $\left[1 s^{2}\right] 2 s^{2} 2 p^{2}, 2 s^{3}$ and $2 p^{4}$ configurations have been calculated by many workers, and have been compared and discussed in detail in the literature. However, similar calculations including the levels of the $2 s^{2} 2 \mathrm{p} 3 \ell$ configurations are rather limited, and radiative rates are available for a few transitions only. Therefore, the present calculations have reported values of oscillator strengths and radiative rates for all allowed transitions among 46 levels of the above six configurations, as well as improving the accuracy of the earlier work. The differences with the earlier calculations are generally within $30 \%$, and the accuracy of our present calculations may not be better than $20 \%$, especially for those transitions whose $f$-values are large $(\geq 0.1)$. For weaker transitions having smaller $f$-values, the corresponding accuracy estimate may be much lower.

Acknowledgements. The work reported in this paper has been financed by the EPSRC and PPARC of the United Kingdom, the National Science Foundation and Division of Chemical Sciences, Office of Basic Energy Sciences, DoE of the United States, and NATO grant CRG 930722.

\section{References}

Aggarwal, K. M. 1998, ApJS, 118, 589

Aggarwal, K. M., Hibbert, A., \& Keenan, F. P. 1997a, ApJS, 108, 393

Aggarwal, K. M., Hibbert, A., Keenan, F. P., \& Norrington, P. H. 1997b, ApJS, 108, 575

Aggarwal, K. M., Keenan, F. P., \& Msezane, A. Z. 2001, ApJS, 136, 763

Cowan, R. D. 1981, The Theory of Atomic Structure and Spectra (Berkeley: Univ. of California Press)

Dyall, K. G., Grant, I. P., Johnson, C. T., Parpia, F. A., \& Plummer, E. P. 1989, Comput. Phys. Commun., 55, 425

Fawcett, B. C. 1987, At. Data Nucl. Data Tables, 37, 367

Hibbert, A. 1975, Comput. Phys. Commun., 9, 141

Kelly, R. L. 1987, J. Phys. Chem. Ref. Data Suppl., 16, 1

Trabert, E. 2002, Phys. Scr., T100, 88

Zhang, H. L., \& Sampson, D. H. 1996, At. Data Nucl. Data Tables, 63,275

Zhang, H. L., \& Sampson, D. H. 1997, At. Data Nucl. Data Tables, 65,183 